\title{
Phase transition in the pairing-plus-quadrupole model
}

\author{
C. Bahri, D. J. Rowe, and W. Wijesundera \\ Department of Physics, University of Toronto, Toronto, Ontario, Canada M5S 1A7
}

(Received 11 May 1998)

\begin{abstract}
We consider the pairing-plus-quadrupole model Hamiltonian within the framework of a many-fermion shell model. The Hamiltonian exhibits a transition from a superconducting to a rotational phase with variation of a parameter. The model has analytical solutions in its two limits due to the presence of dynamical symmetries. However, the symmetries are basically incompatible with one another which means that no simple solution exists in intermediate situations. Exact (numerical) solutions are nevertheless possible on a computer and enable one to study the behavior of competing but incompatible symmetries and the phase transitions that result in a semirealistic situation. This mixed-symmetry dynamics generates a rich structure which gives new insight for the study of phase transitions. [S0556-2813(98)03309-3]

PACS number(s): 21.60.Fw, 03.65.Fd, 05.70.Fh, 21.60.Ev
\end{abstract}

\section{INTRODUCTION}

The problem of understanding superconducting pair correlations in rotational nuclei has been a longstanding challenge for many years [1]. The pairing-plus-quadrupole model (PQM), introduced by Bohr, Mottelson, and Belyaev [2], provides a useful schematic Hamiltonian for investigation of the phenomenon. However, exact shell model calculations for the PQM Hamiltonian are only possible for highly truncated model spaces.

The conventional treatment of the PQM is within the framework of the deformed Hartree-Fock-Bogolyubov (HFB) approximation or, more simply, the Nilsson modelBCS approximation [3,4]. Such quasiparticle generalizations of mean-field theory yield many solutions. Some solutions may correspond to deformed states without pair correlations; others may be spherical or less deformed and exhibit strong pair correlations. For different parameters in the Hamiltonian, different solutions emerge. Furthermore, with variation of the parameters, phase transitions can occur in the solution that lies lowest in energy. This raises a question as to whether such phase transitions are real or artifacts of the HFB approximation. When improvements are made to the HFB approximation by restoring the broken rotational and particle number symmetries by angular momentum and particle number projections, respectively, it appears that the phase transitions are smoothed out [5]. Remnants of them may survive and even become quite sharp in large systems. But without the ability to carry out exact calculations, it is hard to know if the results are to be taken seriously.

An important step in understanding the nature of pair correlations in rotational nuclei was made by Bahri et al. These authors carried out truncated-free shell model calculations for any number of nucleons of a single type in the $2 p 1 f$ shell and for a few nucleons in the $3 s 2 d 1 g$ shell in an SU(3) basis [6-8]. Such calculations are informative and applications to some rare-earth nuclei have been reported within the framework of the pseudo-SU(3) version of the technology [8]. However, our interest is in larger doubly open-shell rareearth nuclei and realistic calculations for such nuclei are impossible. The main obstacle is that the dimensions of the Hilbert space increase exponentially with the particle num- ber. The computational difficulties are reduced in an $\mathrm{SU}(3)$ shell model basis, as compared to traditional calculations in a $j j$-coupled basis, when the interactions are sums of components of a relatively small number of SU(3) tensors. When the Hamiltonian possesses an SU(3) dynamical symmetry, the advantages of an $\mathrm{SU}(3)$ basis are obvious. In fact, there are even greater advantages to expressing shell model wave functions in an $\mathrm{SU}(3)$ basis when a rotational spectrum emerges from the calculations but $\mathrm{SU}(3)$ is not a good dynamical symmetry. One then gains physical insight into the nature of the mixing of SU(3) irreps and the true meaning of adiabaticity of collective motions in group theoretical terms [9].

We present here a model many-fermion system that exhibits both superconducting and rotational phases. The model is sufficiently sophisticated to exhibit complex and competing collective dynamics. It is also simple enough to be amenable to exact numerical solution. The model consists of a system of fermions with intrinsic spin $\kappa-\frac{1}{2}$ (where $\kappa$ is an integer) and orbital angular momentum $l=1$. The fermions interact through a mixture of pairing and $\mathrm{SU}(3)$ quadrupole-quadrupole interactions.

The characteristics of the model are dictated by the fact that the PQM problem can only be solved by algebraic methods within the $1 p$ shell. However, since the number of spin$\frac{1}{2}$ nucleons that can be accommodated within the $1 p$ shell is too small to be of interest, we artificially increase the value of the intrinsic spin to allow a large number of fermions to occupy the shell without violating the Pauli principle. We thereby obtain an exactly solvable model with nontrivial and coexisting rotational and superconducting phases.

Note that in a preliminary introduction of our model [10], we restricted the spin of the fermions to $\frac{1}{2}$ but invoked an extra flavor degree of freedom. However, the result is precisely the same because neither the spin nor the flavor degrees of freedom participate in the dynamics.

The intrinsic spin can be interpreted as a pseudospin as, for example, in Ginocchio's model [11]. Indeed, our model can be interpreted as a special case of the fermion dynamical symmetry model [12]. Furthermore, it contracts to the interacting boson model [13] as $\kappa \rightarrow \infty$.

The model is admittedly contrived. However, we stress 
that our purpose is not to explain the properties of any particular nucleus. It is rather to provide a solvable model to investigate the properties of phase transitions as a prelude to the construction of more realistic models. We recall that the much simpler Lipkin model [14] was of considerable value for such investigations and that it was used to test a number of many-body theories that were subsequently applied with some confidence to realistic situations.

\section{HAMILTONIAN AND MODEL SPACE}

We consider a model with Hamiltonian

$$
H=\alpha H_{1}+(1-\alpha) H_{2},
$$

where $H_{1}$ and $H_{2}$ are, respectively, Hamiltonians for rotational motion and superconductivity; $\alpha$ is a parameter in the range $0 \leqslant \alpha \leqslant 1$. With variation of $\alpha$, the system undergoes a transition from one phase to the other.

A microscopic model for the rotational states of an $A$-particle nucleus is given by a Hamiltonian of the form

$$
H_{\mathrm{RM}}=\frac{1}{2 m} \sum_{n}^{A} p_{n}^{2}+\frac{1}{2} m \omega^{2} \sum_{n}^{A} r_{n}^{2}+V(Q) \text {, }
$$

where $V(Q)$ is a (low order) rotationally invariant polynomial in the quadrupole moments of the nucleus [15]. The building blocks of such a Hamiltonian $\left(\Sigma p_{n}^{2}, \Sigma r_{n}^{2}\right.$, and the components of $Q$ ) are elements of the (noncompact) symplectic algebra $\mathrm{sp}(3, \mathrm{R})$. Consequently, matrix elements of $H_{\mathrm{RM}}$ can be computed in a partially ordered basis by algebraic methods and $H_{\mathrm{RM}}$ is diagonalizable on a computer (to within round-off errors).

Models of superconductivity [16] are given by Hamiltonians with single-particle energies and pairing interactions of the form

$$
H_{\mathrm{SC}}=\sum \varepsilon_{j} a_{j m}^{\dagger} a_{j m}-\sum G_{j j^{\prime}} a_{j m}^{\dagger} a_{j m}^{\dagger} a_{j^{\prime} \bar{m}^{\prime}} a_{j^{\prime} m^{\prime}},
$$

where $\left\{a_{j m}^{\dagger}, a_{j m}\right\}$ are creation and annihation operators for fermions of angular momentum $j$ and its $z$ component $m ; j \bar{m}$ label the time-reverse of the state labeled by $j m$. Such a Hamiltonian has a spectrum generating algebra (SGA) comprising a direct sum of $\mathrm{su}(2)$ (quasispin) algebras. Consequently, it can be diagonalized numerically [17] (if the number of single particle states is not too large).

The actions of the $\operatorname{sp}(3, \mathrm{R})$ and quasispin Lie algebras are incompatible according to the following definition (given in [10]): the actions of two groups (or algebras) are said to be incompatible on a Hilbert space $H$ if they do not commute with one another and if the space $\mathbb{H}$ carries reducible representations of both groups (algebras) but has no proper subspace that is invariant under both groups (algebras). As a result of the incompatibility of the $\operatorname{sp}(3, \mathbb{R})$ and quasispin algebras and the fact that the unitary irreps of $\operatorname{sp}(3, \mathrm{R})$ are of infinite dimension, it is not possible to diagonalize a linear combination of $H_{\mathrm{RM}}$ and $H_{\mathrm{SC}}$ without an unjustified truncation of the space. It is then an interesting challenge to find approximate methods for determining the spectra of such combinations.
To simplify the problem, we restrict the Hamiltonian to a single spherical harmonic oscillator shell $\nu$ for which the number of single particle orbitals is $n=\frac{1}{2}(\nu+1)(\nu+2)$. The microscopic rotor model, then, reduces to the Elliott model for which a suitable Hamiltonian is [18]

$$
H_{1}=-\chi \mathcal{Q} \cdot \mathcal{Q},
$$

where $\mathcal{Q}$ is the $\operatorname{su}(3)$ quadrupole tensor. This Hamiltonian has spectrum given by

$$
E(\lambda \mu J)=-6 \chi C_{2}(\lambda \mu)+3 \chi J(J+1)
$$

where $(\lambda \mu)$ is an $\operatorname{su}(3)$ highest weight, $J$ is the angular momentum, and

$$
C_{2}(\lambda \mu)=\frac{2}{3}\left(\lambda^{2}+\mu^{2}+\lambda \mu+3 \lambda+3 \mu\right)
$$

is the value of the su(3) Casimir invariant.

A simplification of $H_{\mathrm{SC}}$ is achieved by setting all the single-particle energies equal and giving the pair-coupling constants a single value, $G_{j j^{\prime}}=G$, so that, to within an overall constant, the Hamiltonian $H_{\mathrm{SC}}$ reduces to

$$
H_{2}=-G S_{+} S_{-}
$$

where

$$
\begin{gathered}
S_{+}=\sum_{j m>0}(-1)^{j+m} a_{j m}^{\dagger} a_{j,-m}^{\dagger}, \\
S_{0}=\frac{1}{2} \sum_{j m>0}\left(a_{j m}^{\dagger} a_{j m}-a_{j,-m} a_{j,-m}^{\dagger}\right), \\
S_{-}=\sum_{j m>0}(-1)^{j+m} a_{j,-m} a_{j m} .
\end{gathered}
$$

This Hamiltonian, proposed by Kerman et al. [16], has analytical solutions because $S_{ \pm}$are the raising and lowering operators of a single $\operatorname{su}(2)$ algebra with $\left[S_{+}, S_{-}\right]=2 S_{0}$ and $\left[S_{0}, S_{ \pm}\right]= \pm S_{ \pm}$. It has a spectrum given by

$$
E(N, s)=-G\left(s+m_{s}\right)\left(s-m_{s}+1\right),
$$

where $s$ is a quasispin quantum number and $m_{s}$ is an eigenvalue of $S_{0}$. In terms of seniority $v$ and nucleon number $N, s$ is given by $s=\frac{1}{2}(n-v)$, and $m_{s}$ is given by $m_{s}=-\frac{1}{2}(n$ $-N)$. Thus, we obtain the familiar expression for the energy levels

$$
E(N, v)=-\frac{1}{4} G(N-v)(2 n-v-N+2) .
$$

With these simplifications, the dynamical symmetries of $H_{1}$ and $H_{2}$ can be combined. The smallest Lie algebra that contains both the $\mathrm{su}(2)$ and $\mathrm{su}(3)$ algebras, as realized above, is the compact symplectic algebra $\operatorname{sp}(n)$. The Lie algebra $\operatorname{sp}(n)$ (more correctly its complex extension) is spanned by operators 


$$
\begin{aligned}
& A_{i j}=\sum_{m}(-1)^{s+m} a_{i m}^{\dagger} a_{j,-m}^{\dagger}, \\
& C_{i j}=\frac{1}{2} \sum_{m}\left(a_{i m}^{\dagger} a_{j m}-a_{j m} a_{i m}^{\dagger}\right), \\
& B_{i j}=\sum_{m}(-1)^{s+m} a_{i m} a_{j,-m},
\end{aligned}
$$

where $i$ and $j$ run over the range $1, \ldots, n, s$ is the intrinsic spin $\left(s=\kappa-\frac{1}{2}\right)$ and $m$ is summed over the spin values. The operators $\left\{A_{i j}, C_{i j}, B_{i j}\right\}$ satisfy the commutation relations

$$
\begin{aligned}
& {\left[C_{i j}, A_{k l}\right]=\delta_{j k} A_{i l}+\delta_{j l} A_{i k},} \\
& {\left[C_{i j}, B_{k l}\right]=-\delta_{i k} B_{j l}-\delta_{i l} B_{j k},} \\
& {\left[B_{i j}, A_{k l}\right]=\delta_{j k} C_{l i}+\delta_{j l} C_{k i}+\delta_{i k} C_{l j}+\delta_{i l} C_{k j} .}
\end{aligned}
$$

The subset of operators $\left\{C_{i j}\right\}$ span the $\mathrm{u}(n) \subset \operatorname{sp}(n)$ subalgebra and satisfy the commutation relations

$$
\left[C_{i j}, C_{k l}\right]=\delta_{j k} C_{i l}-\delta_{j l} C_{i k}
$$

For $n \geqslant 3, \operatorname{sp}(n)$ contains Elliott's $\operatorname{su}(3)$ and Kerman's $\mathrm{su}(2)$ quasispin algebra, with

$$
S_{+}=\frac{1}{2} \sum_{i} A_{i i}, \quad S_{0}=\frac{1}{2} \sum_{i} C_{i i}, \quad S_{-}=-\frac{1}{2} \sum_{i} B_{i i},
$$

as subalgebras. Thus, $\operatorname{sp}(n)$ is a SGA for the Hamiltonian of Eq. (1).

Note that the actions of the subalgebras su(3) and su(2) of $\operatorname{sp}(n)$ are still incompatible on the Hilbert space for an $\operatorname{sp}(n)$ irrep. And, as a consequence, the problem remains intractable unless $n$ is small. Thus, we consider here the smallest $\operatorname{sp}(n)$ Lie algebra that contains both $\operatorname{su}(3)$ and $\operatorname{su}(2)$, i.e., $\operatorname{sp}(3)$.

With nucleons of spin $\frac{1}{2}$, we now face a problem because, the above realization of $\operatorname{sp}(3)$, with $\kappa=1$, has only irreps that are too small to be of interest; the largest su(3) irrep contained in such an $\mathrm{sp}(3)$ irrep is of highest weight $(2,0)$, as one knows from putting nucleons of one type into the $1 p$ shell. We therefore let $\kappa$ take much larger values, and thereby generate the large $\operatorname{su}(3)$ subirreps of the type expected in heavy deformed nuclei [19].

Matrix elements of the $\operatorname{sp}(n)$ Lie algebra in irreps with lowest and/or highest weights are given by vector coherent state (VCS) theory [20]. Explicit expressions are given in the following section for the irreps of $\operatorname{sp}(3)$ with highest weight $(\kappa, \kappa, \kappa)$ and lowest weight $(-\kappa,-\kappa,-\kappa)$, which are the relevant irreps for present purposes. These are the $\operatorname{sp}(3)$ irreps generated by repeated application of the fermion pair creation operators $\left\{A_{i j}\right\}$ to a fermion vacuum state $|0\rangle$, a state which satisfies the equation

$$
B_{i j}|0\rangle=0, \quad C_{i j}|0\rangle=-\delta_{i j} \kappa|0\rangle .
$$

An orthonormal basis for an $\mathrm{sp}(3)$ irrep of highest weight $(\kappa, \kappa, \kappa)$, which reduces the subalgebra chain

$$
\mathrm{sp}(3) \supset \mathrm{u}(3) \supset \mathrm{su}(3) \supset \mathrm{so}(3) \supset \mathrm{so}(2),
$$

is given by a set of states $\{|n K J M\rangle\}$, where $n$ $\equiv\left\{n_{1}, n_{2}, n_{3}\right\}$, a triple of integers with $n_{1} \geqslant n_{2} \geqslant n_{3}$, is a $\mathrm{u}(3)$ highest weight and $J M$ are the usual $\operatorname{so}(3) \supset \operatorname{so}(2)$ angular momentum quantum numbers; $K$ (associated with the projection of $J$ onto the intrinsic $z$ axis) indexes multiplicities of $J$ in the $\operatorname{su}(3)$ irrep with highest weight $(\lambda, \mu)=\left(n_{1}-n_{2}, n_{2}-n_{3}\right)[18]$.

\section{MATRIX ELEMENTS}

By construction, the Hamiltonian $H_{1}$ is diagonal in the $\mathrm{sp}(3) \supset \mathrm{u}(3) \supset \mathrm{su}(3) \supset \mathrm{so}(3)$ basis. Thus, we focus on the computation of matrix elements of $\mathrm{H}_{2}$, noting that $\mathrm{H}_{2}$ is a quadratic function of elements of the $\mathrm{sp}(3)$ algebra.

The $\operatorname{sp}(3)$ operators $\left\{A_{i j}, B_{i j}, C_{i j}\right\}$ are components of $\mathrm{u}(3)$ tensors. Thus, in the $\mathrm{u}(3)$-coupled basis, their matrix elements are expressible, by means of the Wigner-Eckart theorem, as products of u(3)-reduced matrix elements and su(3) Clebsch-Gordan coefficients. First, observe that the raising operators $\left\{A_{i j}\right\}$ transform as a basis for a $\mathrm{u}(3)$ irrep of highest weight $\{2,0,0\}$. Moreover the spherical tensor combinations $A_{0}$ and $\left\{A_{2 m}\right\}$, defined such that

$$
\begin{aligned}
& A_{0}=\frac{1}{\sqrt{6}} \sum_{i} A_{i i}, \\
& A_{20}=\frac{1}{\sqrt{12}}\left(2 A_{11}-A_{22}-A_{33}\right),
\end{aligned}
$$

transform like $J=0$ and $J=2$ states, respectively. Thus, the matrix elements of $S_{+}=\sqrt{\frac{3}{2}} A_{0}$, for example, are directly inferred from the fact that

$$
\begin{aligned}
& \left\langle n^{\prime} K^{\prime} J^{\prime} M^{\prime}\left|A_{0}\right| n K J M\right\rangle \\
& \quad=\left\langle n^{\prime}\|A\| n\right\rangle\left((\lambda \mu) K J M,(20) 0 \mid\left(\lambda^{\prime} \mu^{\prime}\right) K^{\prime} J^{\prime} M^{\prime}\right),
\end{aligned}
$$

where $\left((\lambda \mu) K J M,(20) 0 \mid\left(\lambda^{\prime} \mu^{\prime}\right) K^{\prime} J^{\prime} M^{\prime}\right)$ is an su(3) CG coefficient and $\lambda=n_{1}-n_{2}, \mu=n_{2}-n_{3}$. Matrix elements of $S_{-}=-\sqrt{\frac{3}{2}} B_{0}$ are similarly inferred by means of the identity

$$
\left\langle n^{\prime \prime}\|B\| n\right\rangle=-\sqrt{\frac{\operatorname{dim}(\lambda \mu)}{\operatorname{dim}\left(\lambda^{\prime \prime} \mu^{\prime \prime}\right)}}\left\langle n\|A\| n^{\prime \prime}\right\rangle,
$$

where $\operatorname{dim}(\lambda \mu)=\frac{1}{2}(\lambda+\mu+2)(\lambda+1)(\mu+1)$ is the dimension of the $\mathrm{SU}(3)$ irrep of highest weight $(\lambda \mu)$.

$\mathrm{U}(3)$-reduced matrix elements of the $A$ operators can be found, for example, in Ref. [21] where it is shown, by means of VCS theory, that

$$
\begin{aligned}
& \left\langle\left\{n_{1}+2, n_{2}, n_{3}\right\}\|A\|\left\{n_{1}, n_{2}, n_{3}\right\}\right\rangle \\
& \quad=\left(\frac{\left(n_{1}+4\right)\left(n_{1}-n_{2}+2\right)\left(n_{1}-n_{3}+3\right)\left(2 \kappa-n_{1}\right)}{2\left(n_{1}-n_{2}+3\right)\left(n_{1}-n_{3}+4\right)}\right)^{1 / 2},
\end{aligned}
$$




$$
\begin{aligned}
& \left\langle\left\{n_{1}, n_{2}+2, n_{3}\right\}\|A\|\left\{n_{1}, n_{2}, n_{3}\right\}\right\rangle \\
& \quad=\left(\frac{\left(n_{2}+3\right)\left(n_{1}-n_{2}\right)\left(n_{2}-n_{3}+2\right)\left(2 \kappa+1-n_{2}\right)}{2\left(n_{1}-n_{2}-1\right)\left(n_{2}-n_{3}+3\right)}\right)^{1 / 2}, \\
& \left\langle\left\{n_{1}, n_{2}, n_{3}+2\right\}\|A\|\left\{n_{1}, n_{2}, n_{3}\right\}\right\rangle \\
& =\left(\frac{\left(n_{3}+2\right)\left(n_{2}-n_{3}\right)\left(n_{1}-n_{3}+1\right)\left(2 \kappa+2-n_{3}\right)}{2\left(n_{1}-n_{3}\right)\left(n_{2}-n_{3}-1\right)}\right)^{1 / 2},
\end{aligned}
$$

with $n_{1}, n_{2}$, and $n_{3}$ running over the ranges

$$
\begin{gathered}
n_{1}=0,2, \ldots, 2 \kappa, n_{2}=0,2, \ldots, n_{1}, \\
n_{3}=0,2, \ldots, n_{2} .
\end{gathered}
$$

The matrix elements of $H_{2}=-G S_{+} S_{-}$can now be evaluated from the matrix elements of $S_{+}$and $S_{-}$by summing over intermediate states. They are evaluated more efficiently, by expressing $S_{+} S_{-}$as a sum of irreducible $\mathrm{SU}(3)$ tensors,

$$
S_{+} S_{-}=-\frac{3}{2}\left[\frac{1}{\sqrt{6}}[A \times B]^{(00)}-\frac{5}{\sqrt{6}}[A \times B]_{0}^{(22)}\right] \text {, }
$$

using the fact that

$$
A_{0} B_{0}=\sum_{\nu=0,2}((20) 0 ;(02) 0 \|(\nu \nu) 0)[A \times B]_{0}^{(\nu \nu)} .
$$

The tensor $[A \times B]^{(00)}$ is an $\mathrm{SU}(3)$ invariant and has only diagonal matrix elements in an $\mathrm{SU}(3)$ basis. The matrix elements of $[A \times B]^{(22)}$ are given by the general expression

$$
\begin{aligned}
\left\langle n^{\prime}\left\|[A \times B]^{(\nu \nu)}\right\| n\right\rangle_{\tau}= & \sum_{n^{\prime \prime}} U\left(\left(\lambda^{\prime} \mu^{\prime}\right)(20)(\lambda \mu)(02) ;\left(\lambda^{\prime \prime} \mu^{\prime \prime}\right)\right. \\
& \left.\times(\nu \nu)_{\tau}\right)\left\langle n^{\prime}\|A\| n^{\prime \prime}\right\rangle\left\langle n^{\prime \prime}\|B\| n\right\rangle,
\end{aligned}
$$

where $U(----;--)$ is an $\operatorname{su}(3)$ Racah coefficient with $\tau$ denoting the outer multiplicity label of the SU(3)-coupling $(\lambda \mu) \times(\nu \nu) \rightarrow\left(\lambda^{\prime} \mu^{\prime}\right)_{\tau}$. The $\operatorname{su}(3)$ Clebsch-Gordan and Racah coefficients are readily computed using the DraayerAkiyama SU(3) package [22].

\section{THE SU(3) MODEL AS A ROTOR MODEL}

It is well known that the Elliott SU(3) model gives spectra that approach those of the rigid rotor model for largedimensional irreps. This result is explained by the fact that the su(3) algebra contracts to a dynamical algebra [23] for the rigid rotor, known as $\operatorname{rot}(3)$, for large values of the su(3) Casimir operator [24]. Thus, there is a natural correspondence between the irreps of $\operatorname{su}(3)$ and those of the rigid rotor.

An irrep of $\operatorname{su}(3)$ is labeled by a highest weight, i.e., a pair of non-negative integers, $(\lambda \mu)$, corresponding to a point in the positive Weyl chamber of weight space. Thus, the inequivalent $\mathrm{su}(3)$ irreps form a lattice in a $60^{\circ}$ segment of weight space.
Irreps of the rigid-rotor algebra $\operatorname{rot}(3)$ are labeled by intrinsic quadrupole moments which are conventionally expressed in terms of $\beta$ and $\gamma$ deformation parameters by

$$
\begin{gathered}
\bar{Q}_{\nu}=k \beta\left[\delta_{\nu 0} \cos \gamma+\frac{1}{\sqrt{2}}\left(\delta_{\nu 2}+\delta_{\nu,-2}\right) \sin \gamma\right], \\
\beta \geqslant 0, \quad 0 \leqslant \gamma<60^{\circ},
\end{gathered}
$$

where $k$ is a normalization constant. Thus, the irreps of $\operatorname{rot}(3)$ correspond to a continuous set of points in a $60^{\circ}$ segment of a space which can be identified with the su(3) weight space.

The correspondence between $(\beta, \gamma)$ and $(\lambda, \mu)$ was given by considering the contraction limit [24] and, more precisely, by comparing $\mathrm{su}(3)$ and $\operatorname{rot}(3)$ matrix elements $[25,26,7]$. It is given by

$$
\begin{aligned}
& k \beta \cos \gamma=2 \lambda+\mu+3, \\
& k \beta \sin \gamma=\sqrt{3}(\mu+1) .
\end{aligned}
$$

Thus, the essential difference between the $\mathrm{su}(3)$ and $\operatorname{rot}(3)$ algebras is that the highest weights of $\operatorname{su}(3)$ take discrete values whereas the corresponding $\operatorname{rot}(3)(\beta, \gamma)$ values are continuous. However, for states of angular momentum $J \ll \lambda+\mu$, the distinction becomes less and less important as $\lambda+\mu \rightarrow \infty$. Thus, the close correspondence between the two algbraic models makes it possible to interpret the wave functions in a mixed-su(3) shell-model calculation in terms of $\beta$ and $\gamma$ vibrational fluctuations.

\section{RESULTS AND DISCUSSIONS}

The energy spectra can be obtained by diagonalizing the matrix of the Hamiltonian of Eq. (1). In this paper, we restrict consideration to the yrast states which exhibit the effects we wish to explore. To examine the competition between the $\mathrm{SU}(2)$ and $\mathrm{SU}(3)$ dynamical symmetries in these states, we compare the expectation values $\left\langle J\left|C_{2}\right| J\right\rangle$ and $\left\langle J\left|S_{+} S_{-}\right| J\right\rangle$ for the lowest yrast states. The former is a measure of the SU(3) content of the states and the latter indicates the magnitude of the pair correlations.

In the previous paper [10], we set $N=2 \kappa$ and considered a range of $\kappa$ values. We found that, with the coupling constants assigned the values $G=0.05$ and $\chi=0.005$ (in units of energy), remarkably sharp phase transitions occur at a critical value of $\alpha=\alpha_{0} \approx 0.57$. In this paper, we fix $\kappa$ at $\kappa=20$ but consider a range of $N$ values. Some results are shown in Fig. 1 for $N=32,40$, and 48 . The $N=2 \kappa$ results (corresponding to a $\frac{1}{3}$-filled shell) are seen to be special in that they exhibit a much sharper phase transition than obtained for neighboring values of $N$.

Still more remarkable is the rapidity with which the energy levels, for all $N$ values, approach the $J(J+1)$ energies of a rotor once $\alpha$ exceeds the critical value $\alpha_{0} \approx 0.57$. A good indication of this is the fact that the expectation values of both $\left\langle J\left|C_{2}\right| J\right\rangle$ and $\left\langle J\left|S_{+} S_{-}\right| J\right\rangle$ take almost $J$-independent values. Note that, since the Hamiltonian differs from a linear combination of $C_{2}$ and $S_{+} S_{-}$by $3 \alpha \chi \mathbf{J}^{2}, J$-independent val- 

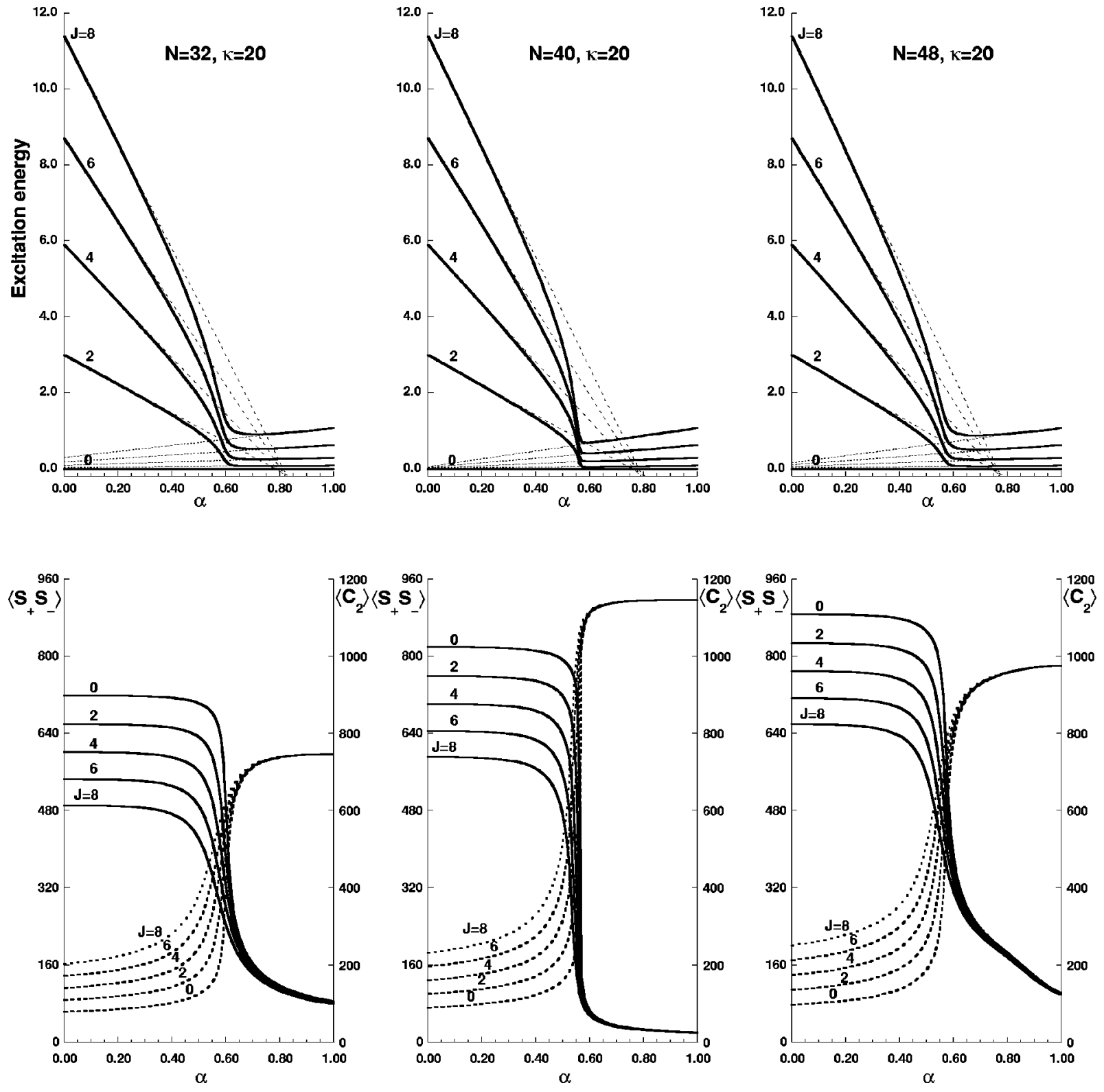

FIG. 1. The upper plots show the excitation energies for different values of $N$ and $\kappa=20$. (The dashed lines are for the first order of $H_{1}$ and the dotted lines are for that of $H_{2}$.) The lower plots show the expectations $\left\langle S_{+} S_{-}\right\rangle$(solid lines) and $\left\langle C_{2}\right\rangle$ (dashed lines) as functions of $\alpha$ for the corresponding $N$ values.

ues of $\left\langle J\left|C_{2}\right| J\right\rangle$ and $\left\langle J\left|S_{+} S_{-}\right| J\right\rangle$ imply energy level differences given by $3 \alpha \chi J(J+1)$.

For $N=40,\left\langle J\left|C_{2}\right| J\right\rangle$ and $\left\langle J\left|S_{+} S_{-}\right| J\right\rangle$ rapidly approach their common $\alpha=1$ values with increasing $\alpha>\alpha_{0}$ indicating that, beyond the critical point, the $N=40$ states belong to essentially unmixed SU(3) irreps. In contrast, the corresponding quantities for $N=32$ and 48 become close to one another, but approach their $\alpha=1$ values much less rapidly. The large slope of the $\left\langle J\left|S_{+} S_{-}\right| J\right\rangle$ curves for $N=48$, for example, is a clear indication that, although the spectra have a rotational $J(J+1)$ character, they correspond, in fact, to highly mixed $\mathrm{SU}(3)$ states.

Thus, the results show that, for some $N$ values, the model exhibits very strong pairing correlations for a range of coupling constants. Nevertheless, in spite of the resulting large mixing of SU(3) representations, the rotational structure, that is normally associated with an unmixed SU(3) situation, manages to survive. In physical terms, the corresponding states are appropriately described as those of a superconducting rotor.

To exhibit the nature of superconducting rotor states, we show in Figs. 2 and 3 the amplitude coefficients of their wave functions in an $\mathrm{SU}(3)\{(\lambda \mu) K J\}$ basis for $N=40$ and $N=48$ and a range of $\alpha$ values in the vicinity of $\alpha_{0}$. The coefficients are shown as histograms for $J=0, \ldots, 8$. It can be seen from Fig. 2 that, for $N=2 \kappa=40$, there is a large mixing of SU(3) irreps for small values of $\alpha$ which decreases rapidly as $\alpha$ passes through the critical region to a much less mixed situation. In contrast, the transition for $N=48$ occurs much less rapidly.

Particularly interesting is the nature of the mixing of $\mathrm{SU}(3)$ irreps in the superconducting rotor regions. It can be seen clearly that, while the amplitude coefficients for states within the transition region have nonzero values for many 

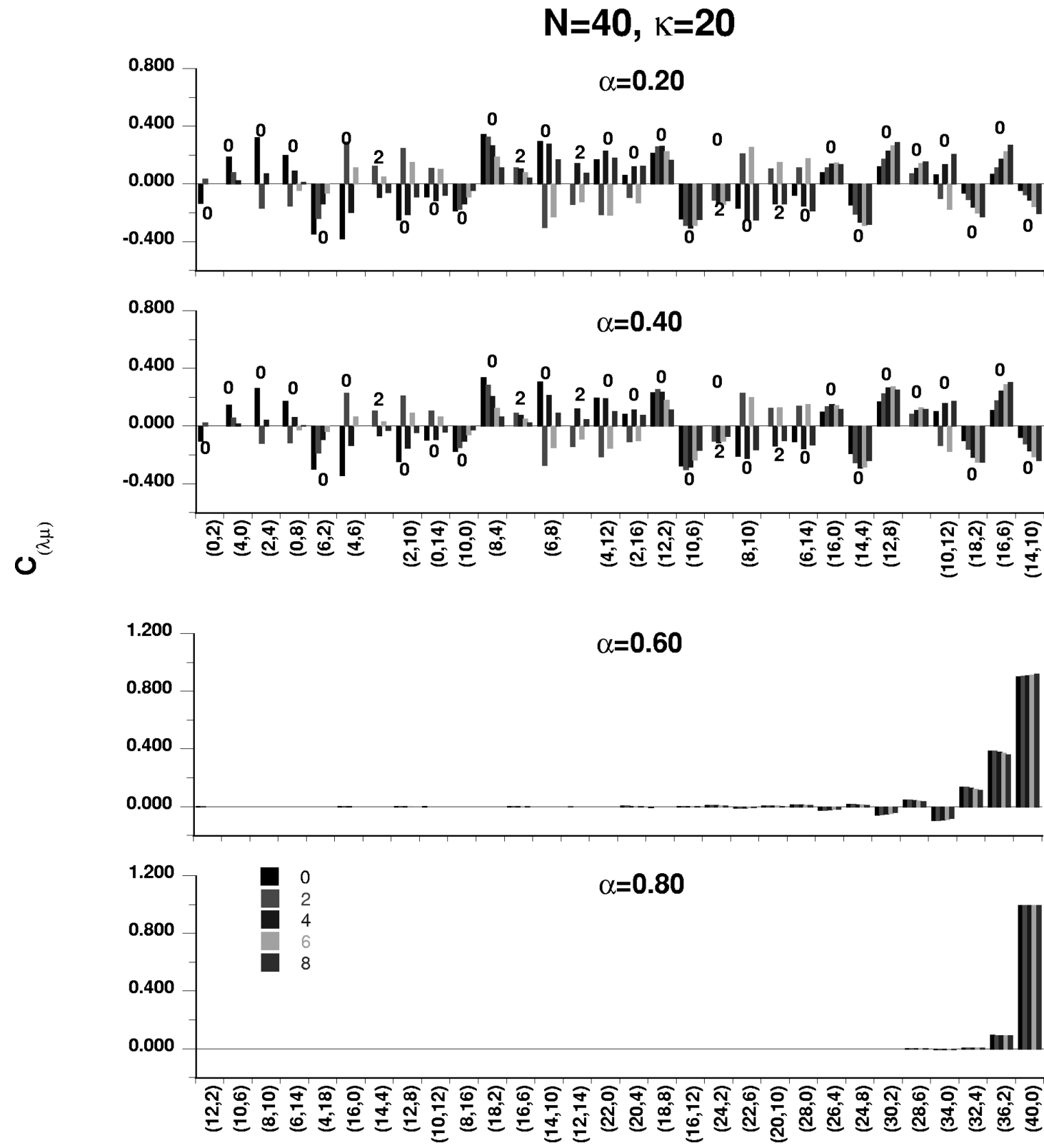

$(\lambda \mu)$

FIG. 2. Histograms of the $\mathrm{SU}(3)$ amplitudes for the yrast states of $N=40$. The upper two plots correspond to superconducting phases and the lower two plots to rigid rotors. Only the 30 largest contributions from a total of 127 irreps are shown on each plot. In the upper two plots, the $K$ values are given right above the histograms. In the lower two plots only the $K=0$ amplitudes are nonzero.

SU(3) irreps, their values are essentially independent of the angular momentum. Moreover, the amplitudes are nonnegligible only for the $K=0$ components of each SU(3) irrep, as shown in Fig. 3. Thus, the eigenstates of the system in these regions have expansions

$$
\left|\psi_{K J M}\right\rangle=\sum_{(\lambda \mu)}\left|\phi_{(\lambda \mu) K J M}\right\rangle C_{(\lambda \mu) K J} \approx \sum_{(\lambda \mu)}\left|\phi_{(\lambda \mu) K J M}\right\rangle C_{(\lambda \mu) K},
$$

with (essentially) $J$ independent expansion coefficients. This means that the concept of a common $K=0$ intrinsic state for each state of the yrast band is remarkably well defined for $\alpha>\alpha_{0}$. The intrinsic states for the superconducting rotors are not the highest weight states of a single SU(3) irrep; they are linear combinations with coefficients determined by the pairing correlations. In group theoretical terms, the $\left|\psi_{K J M}\right\rangle$ states span an adiabatic representation of $\mathrm{su}(3)$ (or more precisely an embedded representation) which is defined to be the weighted average of a number of true su(3) irreps [9]. The characteristic of an embedded SU(3) irrep is that the expansion coefficients $\left\{C_{(\lambda \mu) K J}\right\}$ are independent of $J$; they could, in principle, depend on $K$ but, in fact, it turns out that $K$ $=0$ is a rather good quantum number for the yrast states shown.

Adiabatic (embedded) representations occur naturally for Lie algebras whose matrix elements depend at most linearly on the parameters of their irreps. For SU(3) the matrix elements have an approximate linear dependence on $\lambda$ and $\mu$ for large values of the parameters. Thus, for large dimen- 

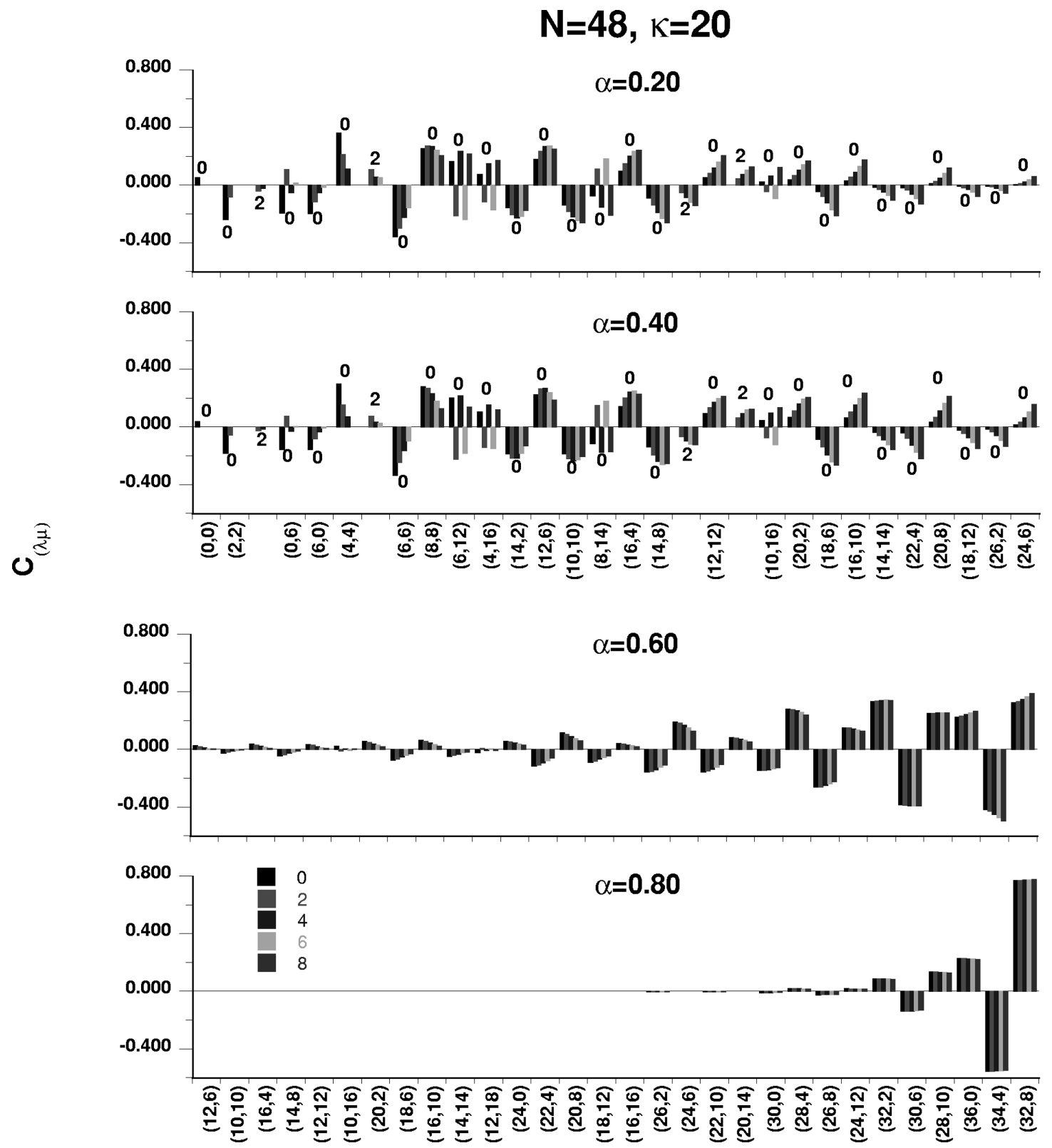

$(\lambda \mu)$

FIG. 3. Histograms of the $\mathrm{SU}(3)$ amplitudes for the yrast states of $N=48$. The upper two plots correspond to superconducting phases and the lower two plots to rotors. Notice that $\alpha=0.60$ corresponds to a superconducting rotor. Only the 30 largest contributions from a total of 177 irreps are shown on each plot. In the upper two plots, the $K$ values are given right above the histograms. In the lower two plots only the $K=0$ amplitudes are nonzero.

sional representations of $\mathrm{SU}(3)$, one can have approximate adiabatic representations. Since the matrix elements of physical operators in such adiabatic representations are the averages of those in true representations, the system is said to have quasidynamical symmetry. Thus, whereas for $\alpha=1$ we have $\mathrm{SU}(3)$ as a genuine dynamical symmetry, for smaller values of $\alpha \geqslant \alpha_{0}$, we have quasi-SU(3) dynamical symmetry, as shown in Fig. 3.

It is especially instructive to see the amplitudes plotted on a weight diagram which, as observed above, has a direct interpretation in terms of a $\beta-\gamma$ deformation plot. The plots are given in Fig. 4 for the coefficients given as histograms in Figs. 2 and 3. By plotting the coefficients in this way, one sees that the superconducting states have a broad distribution of shapes while the rotors have much more well-defined intrinsic shapes; the superconducting rotors have relatively well-defined shapes but with $\beta$ and $\gamma$-vibrational fluctuations.

A useful (but arbitrary) characterization of the transition from a superconductor to a rigid rotor is given by defining a state to be superconducting if $\left\langle S_{+} S_{-}\right\rangle_{\alpha} /\left\langle S_{+} S_{-}\right\rangle_{0}>0.5$ and to be a rigid rotor state if $\left\langle C_{2}\right\rangle_{\alpha} /\left\langle C_{2}\right\rangle_{0}>0.97$. States for which $\left\langle S_{+} S_{-}\right\rangle_{\alpha} /\left\langle S_{+} S_{-}\right\rangle_{0}<0.5$ and $\left\langle C_{2}\right\rangle_{\alpha} /\left\langle C_{2}\right\rangle_{0}<0.97$ are then said to be superconducting rotors. The $N-\alpha$ phase diagram defined according to these criteria is shown in Fig. 5 for $\kappa=20$. This figure exhibits the narrowness of the phase transition between the superconducting and rigid rotor extremes when $N=2 \kappa$, corresponding to a $\frac{1}{3}$-filled shell. The 


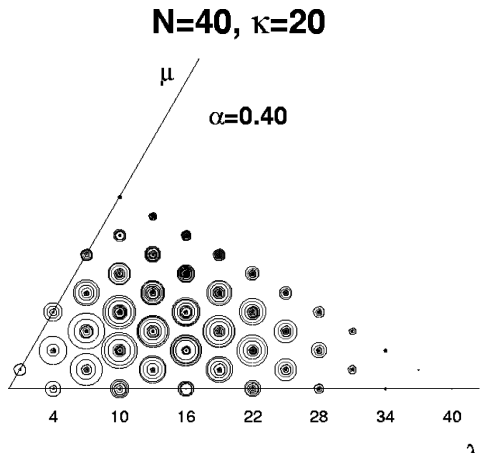

(a)

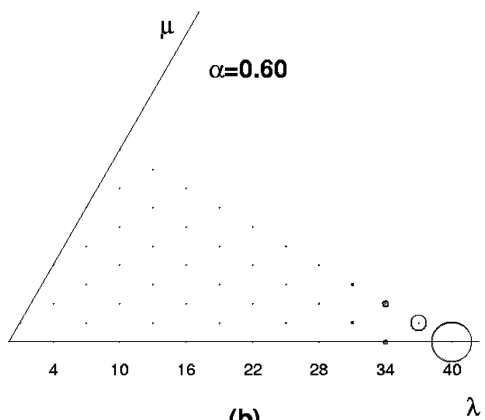

(b)

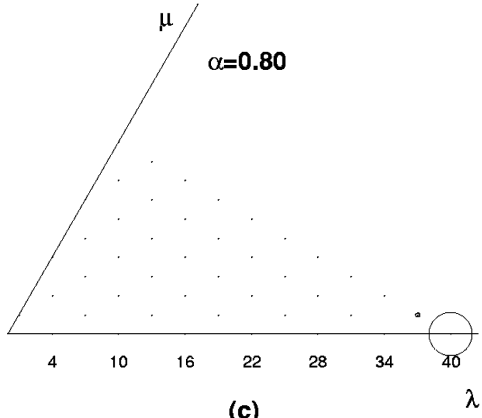

$\mathrm{N}=48, \kappa=20$

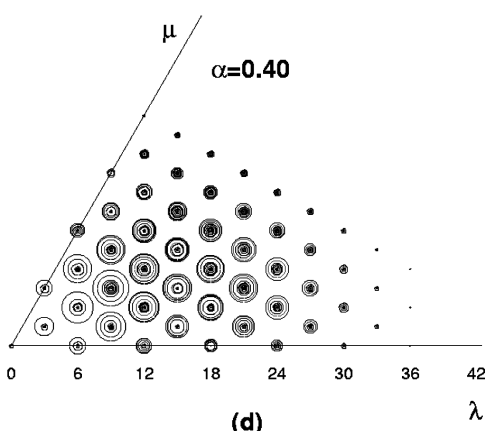

(d)
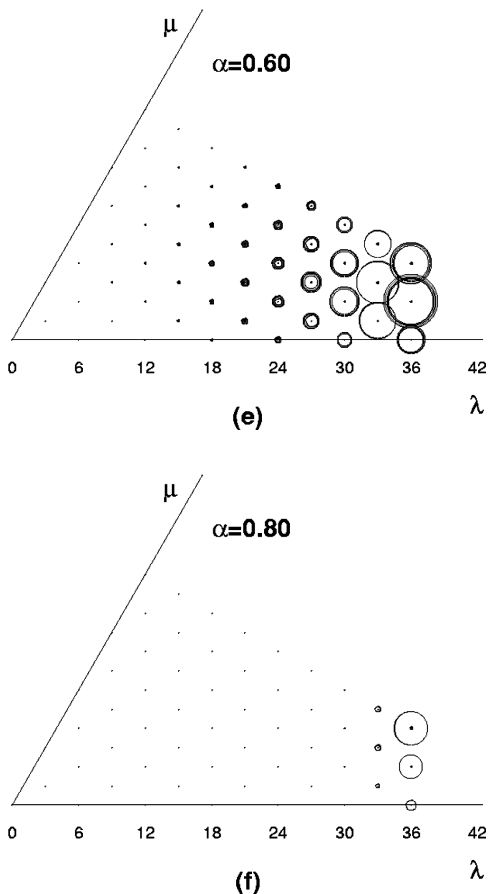

FIG. 4. Weight space representations of the SU(3) amplitudes shown as histograms in Figs. 2 and 3. The contribution of each SU(3) irrep is plotted as a circle of radius proportional to the amplitude at the point in the lattice corresponding to the highest weight of the irrep. The amplitudes for states of angular momentum $J=0,2,4,6$, and 8 are shown as concentric circles. The amplitudes for $\alpha=0.20$ are not shown as they are similar to those for $\alpha=0.40$; (a) and (d) correspond to superconducting phases; (e) to a superconducting rotor; and (b), (c), and (f) to rigid rotors. The scale of the coefficients for (b), (c), and (f) is a factor 2.5 smaller than the rest.

sharpness of the phase transition at $N=2 \kappa$ can be attributed to the Pauli exclusion principle as a result of which a maximum prolate deformation can be achieved when particles fill one third of the shell.

Although we make no attempt to fit experimental data with the model, it is, nevertheless, of interest to observe the remarkable similarity between the experimental energy levels of the Hf-isotopes $(Z=72)[27]$, shown in Fig. 6, and those of the model. In the spirit of simplicity, the model results shown in the figure are for the same $\kappa=20$ and $\chi / G=0.1$ ratio as used previously; thus, the phase diagram in Fig. 5 remains applicable without scale adjustment. We simply adjusted the overall strength of the interaction to fit the energy of the first excited $2^{+}$states of ${ }^{176} \mathrm{Hf}$. The $A$ $=176$ isotope was chosen for the adjustment because it has the lowest first excited $2^{+}$energy level among the Hf isotopes. We also identified this isotope with the $\frac{1}{3}$-filled shell of the model, ignoring the fact that ${ }_{72}^{176} \mathrm{Hf}$ is at the midshell for neutrons in the conventional shell model. The solid lines in Fig. 6 belong to the model with different parameter strengths. The upper (lower) lines are for $\alpha=0.64, G$ $=72.4 \mathrm{keV}$, and $\chi=7.24 \mathrm{keV}(\alpha=0.65, G=73.3 \mathrm{keV}$, and $\chi=7.33 \mathrm{keV}$ ), respectively. The figure shows a close similarity between the energy levels of the model and those of isotopes in the $A=158-184$ range; the similarity breaks down outside of the 158-184 range as exhibited by the energies, indicated by arrows, of the $J=2$ (square) and $J=8$ (triangle) states of the $A=154$ isotope. Note that reliable excitation energies for $A=156$ are not presently available.

\section{CONCLUDING REMARKS}

A study of superconducting effects in real rotational nuclei might consider a pairing plus quadrupole Hamiltonian for spin 1/2 neutrons and protons within an irreducible $\operatorname{Sp}(n)$ subspace of a nuclear valence shell. However, such model calculations are difficult to carry out in all but the smallest shells. They are easy for $n=3$, corresponding to nucleons in 


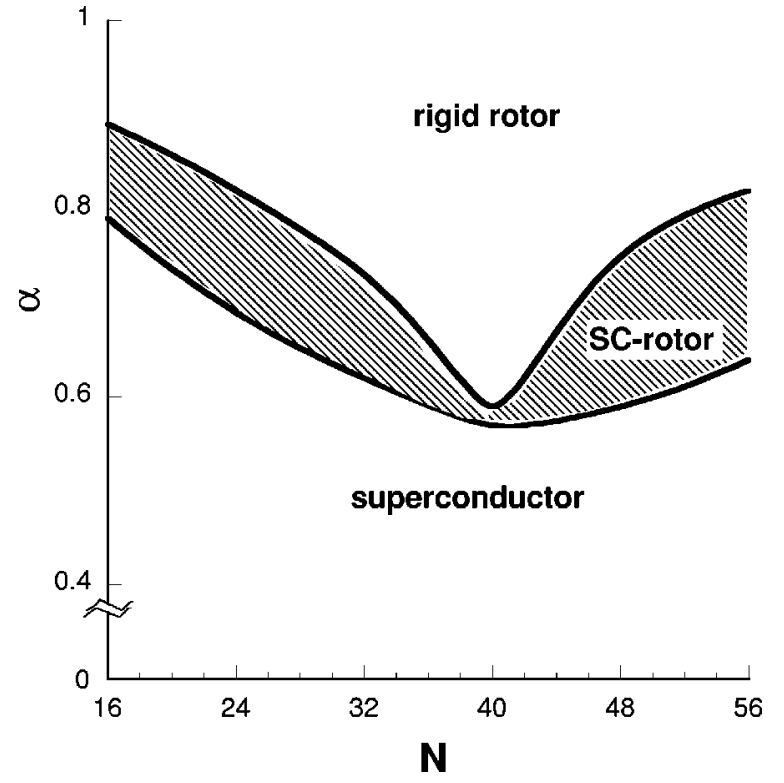

FIG. 5. Phase diagram for the $\mathrm{Sp}(3)$ model parametrized by $\alpha$ and $N$. The shaded area shows a smooth transition from a superconducting to a rotational phase; the transition region is regarded as being in a superconducting rotor phase.

a $1 p$ shell, but already challenging for $n=6$ as required for the $2 s 1 d$ shell. At this time, they are essentially impossible for more than a few particles when $n>3$. However, if they could be done, we believe the results would parallel those obtained in our simple $\mathrm{Sp}(3)$ model. Thus, it would be eminently worthwhile to check the validity of this belief, if only in a few special cases. For, if indeed one were to find adiabatic rotors with strong pairing correlations of the type found in our simple model, then, in carrying out calculations for a real nucleus, for which rotational states are observed experimentally, one would be justified in restricting the calculations in an $\mathrm{SU}(3)$ basis to just the $J=0$ states and in making the assumption that the other states of the yrast band have the same $J$-independent coefficients.

We believe that this is, in fact, what one would find. Indeed, before our model calculations were done, such a result was predicted (cf. Carvalho et al. [24] and Rowe, Rochford, and Repka [9]) to explain the persistence of observed rotational bands in situations where spin-orbit and pairing correlations are expected to be large. Recall that the criterion for the occurrence of rigidlike rotational bands has long been understood to be the adiabaticity of the rotational motions. The intrinsic states of a microscopic rotor have many degrees of freedom and modes of excitation. Thus, the criterion for the appearance of rigidlike rotational bands is only that the coupling between the rotational and intrinsic degrees of freedom is small. And this coupling is small if the rotational angular velocity is small.

One knows that nuclear Hamiltonians are invariant under both translations and rotations. However, while acceptable Hamiltonians should also be invariant under Galilean transformations (center-of-mass momentum boosts), there is no rotational analog of Galilean invariance. This is because a rotating frame of reference is not an inertial frame; there are centrifugal and Coriolis forces. Nevertheless, if the rotational motions are sufficiently slow (adiabatic), a rotating frame

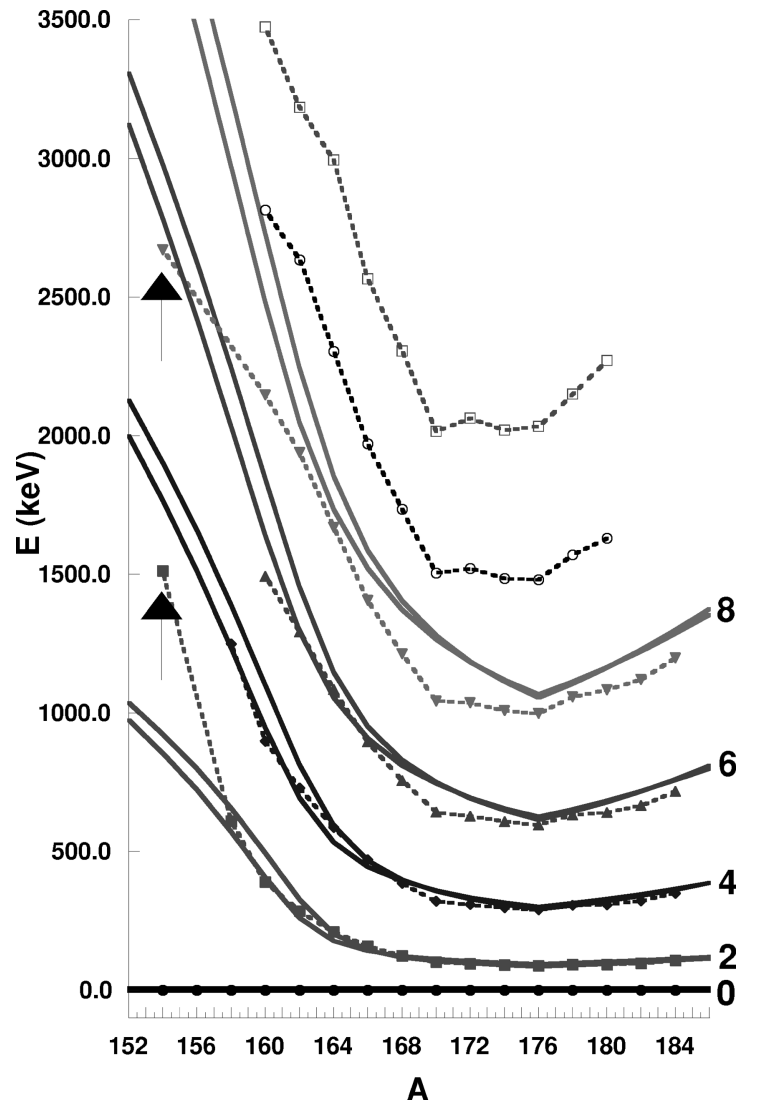

FIG. 6. Comparison between the $S p(3)$ model and experimental energy levels for the Hf isotopes $(Z=72)$. Different experimental points are connected with dashed guiding lines. The two families of solid lines are from the model with different parameter strengths (see text).

approximates an inertial frame. This is clearly what is happening in the so-called superconducting rotor states of our model.

At an abstract algebraic level, the concept of embedded (i.e., adiabatic) rotor [or $\mathrm{SU}(3)$-rotor] representations seems strange. However, our model calculations show that they provide an interesting example of how the physics of a situation can usefully guide the mathematical analysis. For while, from algebraic and computational points of view, the problem of combining two incompatible dynamical symmetries, in all but the smallest model spaces, is a formidable task, the physics of the situation gives hope for very good, even if approximate, solutions.

It is also useful to consider the mixing of SU(3) and rotor irreps from another point of view. Recall that any interaction that commutes with a group of transformations can only mix equivalent representation of that group. Thus, if the infinitesimal generators of the dynamical group of a rotor consist of angular momentum operators and quadrupole moments, then any interaction that is rotationally invariant and commutes with the quadrupole moments is invariant under the dynamical group of the rotor. This implies that while shortrange (momentum-independent) interactions can change the intrinsic states of a rotor dramatically, they can only do so by mixing states of equivalent representations. This is an important result and it is worthwhile to be clear as to its meaning. It means that a short-range interaction should not change 
TABLE I. The amplitude probability coefficients of $(40,0)$ subirrep for the lowest eigenstates, $C_{n 1}$ with $J=0,2,4,6$, and 8 in $\kappa=20, N=40$ configuration. The corresponding probability coefficients, $\left|C_{n 1}\right|^{2}$, are written underneath. At $\alpha=0.57$ a phase transition occurs from superconducting states to rotational ones. The largest components from other (not necessarily the same) subirreps are put in parentheses.

\begin{tabular}{|c|c|c|c|c|c|}
\hline$\alpha \backslash J$ & 0 & 2 & 4 & 6 & 8 \\
\hline \multirow[t]{2}{*}{0.00} & 0 & 0 & 0 & 0 & 0 \\
\hline & 0 & 0 & 0 & 0 & 0 \\
\hline \multirow[t]{2}{*}{0.51} & $0.001(0.294)$ & $0.003(-0.278)$ & $0.011(0.289)$ & $0.036(0.301)$ & $0.097(-0.345)$ \\
\hline & $0.000(0.087)$ & $0.000(0.077)$ & $0.000(0.084)$ & $0.000(0.091)$ & $0.009(0.119)$ \\
\hline \multirow[t]{2}{*}{0.53} & $0.003(-0.285)$ & $0.014(0.273)$ & $0.057(0.289)$ & $0.165(-0.325)$ & $0.346(0.417)$ \\
\hline & $0.000(0.081)$ & $0.000(0.074)$ & $0.003(0.084)$ & $0.027(0.106)$ & $0.119(0.174)$ \\
\hline \multirow[t]{2}{*}{0.55} & $0.028(-0.263)$ & $0.175(-0.259)$ & $0.435(0.425)$ & $0.604(0.502)$ & $0.702(0.516)$ \\
\hline & $0.001(0.069)$ & $0.031(0.067)$ & $0.189(0.180)$ & $0.365(0.252)$ & $0.493(0.266)$ \\
\hline \multirow[t]{2}{*}{0.57} & $0.759(0.486)$ & $0.775(0.486)$ & $0.797(0.481)$ & $0.821(0.470)$ & $0.844(0.455)$ \\
\hline & $0.575(0.236)$ & $0.601(0.236)$ & $0.635(0.231)$ & $0.674(0.221)$ & $0.713(0.207)$ \\
\hline \multirow[t]{2}{*}{0.59} & $0.877(0.420)$ & $0.880(0.417)$ & $0.885(0.411)$ & $0.893(0.402)$ & $0.903(0.389)$ \\
\hline & $0.759(0.177)$ & $0.774(0.174)$ & $0.784(0.169)$ & $0.798(0.161)$ & $0.815(0.151)$ \\
\hline \multirow[t]{2}{*}{1.00} & 1 & 1 & 1 & 1 & 1 \\
\hline & 1 & 1 & 1 & 1 & 1 \\
\hline
\end{tabular}

intrinsic quadrupole moments and $E 2$ transition rates of a rotor band. However, without a dynamical theory of what properties of the flows determine the moments of inertia or without making some specific (e.g., cranking) model assumptions, it is not possible to say what effects the mixing might have on moments of inertia. It should also be understood that the ordering of rotational bands in a situation where many representations occur may be rearranged as a result of pairing correlations so that, with the inclusion of pairing, the representation which best describes the ground state band may very well be different.

We recognize that the schematic pairing force used within an $\operatorname{Sp}(n)$ irrep is not $\mathrm{SU}(3)$ invariant. However, to the extent that the SU(3) model approximates a rotor and the schematic force approximates a short-range interaction, we should expect the latter, if not an SU(3) scalar, to be a low-rank SU(3) tensor. It is known that matrix elements of the SU(3) quadrupole moments vary smoothly with $\lambda$ and $\mu$. Thus, we describe two $S U(3)$ irreps as being similar if their highest weights $(\lambda \mu)$ have similar values. We then conclude that, while a schematic force may mix inequivalent $\mathrm{SU}(3)$ irreps, it will only directly couple irreps which are similar. Observe that the spin-orbit force is a $(1,1) \mathrm{SU}(3)$ tensor so that, although we have not considered a spin-orbit interaction in this paper, we may expect that it too will have similar consequences (cf., results of Rochford and Rowe [9] on spin-orbit mixing).

In spite of the above observations, it should be understood that even though a short-range interaction may commute with the dynamical group of a rotor, it may still result in nonadiabatic effects. Consider a situation in which the energy levels of two equivalent irreps cross. One can imagine, for example, a situation where the ground state band is highly superconducting with relatively small moments of inertia whereas an excited band has smaller pair correlations and larger moments of inertia. At some angular momentum, the yrast band will flip from the lower to the excited band. And, at the crossing angular momentum, one expects large mixing of the two bands, especially if they have similar quadrupole moments and belong to similar irreps of the rotor algebra.

One also knows that the Coriolis force acts on a rotating superconductor in much the same way as a magnetic field acts on a superconductor in condensed matter. Thus, just as a magnetic field suppresses superconductivity, one expects pair correlations to be suppressed due to the Coriolis force beyond some angular momentum value. This effect was noted by Mottelson and Valatin [28] who suggested that a similar collapse of the superconducting pair correlations might be found in rotational nuclei. In fact, it appears that the breaking up of pairs occurs sequentially rather than in a single step. One can see such an effect in Table I, which shows the coefficients of the wave functions in an $\mathrm{SU}(3)$ expansion. One sees that, for values of $\alpha>\alpha_{0}$, the component of a single $\mathrm{SU}(3)$ irrep is more dominant when $J$ is large than when it is small. We anticipate that, for a very large value of $\kappa$ and a correspondingly large value of $N$, we would see a sudden breakdown of the superconductivity at some critical value of the angular momentum and an effect in closer parallel with the Meissner effect. Indeed, it will be very interesting to explore the results of our model in the large $\kappa$ and $N$ limits.

We have stressed that an important reason for constructing a solvable model is to be able to test the validity of 
many-body approximates in an exactly solvable situation. In this context, we note that the conventional many-body description of pair correlations and rotations is within the framework of the deformed Hartree-Fock-Bogolyubov approximation. But, because of the lack of rotational invariance and the violation of number conservation of this approximation, one cannot be sure which HFB results are valid and which are generated by the variational constraints of the approximation. Thus, it will be of considerable interest to see if the onset of deformation with increasing $\alpha$ and the subsequent suppression of superconductivity at still higher values of $\alpha$ are described well in the HFB approximation. If the HFB approximation is successful in the context of the model, it is, of course, no guarantee that it will be reliable in more general situations. However, if it does not work well for the model, it cannot be trusted elsewhere.

Also of interest is the so-called random-phaseapproximation and quasiparticle RPA. It can be seen that the way in which the excitation energies of the first $2^{+}$state in our model drops precipitously with increasing $\alpha$ just prior to the phase transition is reminiscent of RPA (random phase approximation) results for quadrupole vibrational excitation energies. As shown by Thouless [29], the collapse of an RPA vibrational energy to zero indicates an instability of the Hartree-Fock (or HFB) ground state against quadrupole vibrational fluctuations and the expectation of a phase transition to a deformed state. The present model is clearly amenable to analyses in this way.

In conclusion, we note that the philosophy of the RPA, as expressed in the equations of motion formalism [30], is very similar to that of a quasidynamical symmetry. The philosophy of the equations-of-motion formalism is that while eigenstates of the Hamiltonian may be complicated, the relationships between states, particularly if one is regarded as a collective excitation of the other, may be relatively simple. Thus, while it may be quite unreasonable to describe energy eigenstates as independent-particle states, it may be appropriate to approximate excitation operators as one-body operators. In a similar way, we find, for a superconducting rotor, that it is a poor approximation to assume (the valence shell components of) its states to be unmixed $\mathrm{SU}(3)$ states. However, it is a much better approximation to assume that the relationships between the states are described by SU(3) operators. Indeed, this is the meaning of a quasidynamical symmetry. Thus, the use of a quasidynamical symmetry is akin to a field theoretic approach to spectroscopy, in which one focuses on the operators and algebraic equations of motion in preference to the wave functions.

Clearly the model we have constructed has a rich structure and opens up possibilities for the investigation of many interesting phenomena and many-body approximations.

\section{ACKNOWLEDGMENTS}

This investigation was supported by the Natural Sciences and Engineering Research Council of Canada. The authors thank J. Repka and J. L. Wood for fruitful discussions.
[1] D. J. Rowe, Prog. Part. Nucl. Phys. 37, 265 (1996).

[2] A. Bohr, B. R. Mottelson, and D. Pines, Phys. Rev. 110, 936 (1958); S. T. Belyaev, Mat. Fys. Medd. K. Dan. Vidensk. Selsk. 31, 11 (1959).

[3] A. Bohr and B. R. Mottelson, Nuclear Structure (Benjamin, Reading, 1975).

[4] D. J. Rowe, Nuclear Collective Motion (Methuen, London, 1970).

[5] P. Ring and P. Schuck, The Nuclear Many-body Problem (Springer, New York, 1980), Chap. 11.

[6] C. Bahri, Ph.D. thesis, Louisiana State University, 1994.

[7] C. Bahri, J. Escher, and J. P. Draayer, Nucl. Phys. A592, 171 (1995).

[8] D. Troltenier, C. Bahri, and J. P. Draayer, Nucl. Phys. A586, 53 (1995); A589, 75 (1995); D. Troltenier, C. Bahri, J. Escher, and J. P. Draayer, Z. Phys. A 354, 125 (1996).

[9] D. J. Rowe, P. Rochford, and J. Repka, J. Math. Phys. 29, 572 (1988); P. Rochford and D. J. Rowe, Phys. Lett. B 210, 5 (1988).

[10] D. J. Rowe, C. Bahri, and W. Wijesundera, Phys. Rev. Lett. 80, 4398 (1998).

[11] J. N. Ginocchio, Ann. Phys. (N.Y.) 126, 234 (1980).

[12] C-L. Wu, D. H. Feng, and M. Guidry, Adv. Nucl. Phys. 21, 227 (1994).

[13] F. Iachello and A. Arima, The Interacting Boson Model (Cambridge, Cambridge, 1987); D. Bonatsos, Interacting Boson Models of Nuclear Structure (Oxford, Oxford, 1988).
[14] H. J. Lipkin, N. Meshkov, and A. J. Glick, Nucl. Phys. 62, 188 (1965); J. C. Parikh and D. J. Rowe, Phys. Rev. 175, 1293 (1968).

[15] G. Rosensteel and D. J. Rowe, Phys. Rev. Lett. 38, 10 (1977); Ann. Phys. (N.Y.) 126, 343 (1980); P. Park et al., Nucl. Phys. A414, 93 (1984); D. J. Rowe, Rep. Prog. Phys. 48, 1419 (1985); C. Bahri and D. J. Rowe, in preparation.

[16] A. K. Kerman, Phys. Rev. 120, 300 (1961); A. K. Kerman, R. D. Lawson, and M. W. Macfarlane, Phys. Rev. 124, 162 (1961).

[17] H. Chen, T. Song, and D. J. Rowe, Nucl. Phys. A582, 181 (1995).

[18] J. P. Elliott, Proc. R. Soc. London, Ser. A 245, 128 (1958), ibid. 245, 562 (1958); J. P. Elliott and M. Harvey, ibid. 272, 557 (1963).

[19] M. Jarrio, J. L. Wood, and D. J. Rowe, Nucl. Phys. A528, 409 (1991).

[20] D. J. Rowe, J. Math. Phys. 25, 2662 (1984); D. J. Rowe, G. Rosensteel, and R. Carr, J. Phys. A 17, L399 (1984); D. J. Rowe and J. Repka, J. Math. Phys. 32, 2614 (1991).

[21] D. J. Rowe, B. B. Wybourne, and P. H. Butler, J. Phys. A 18, 939 (1985).

[22] J. P. Draayer and Y. Akiyama, J. Math. Phys. 14, 1904 (1973); Y. Akiyama and J. P. Draayer, Comput. Phys. Commun. 5, 405 (1973).

[23] H. Ui, Prog. Theor. Phys. 44, 153 (1970).

[24] J. Carvalho, Ph.D. thesis, University of Toronto (1984); J. Car- 
valho, R. Le Blanc, M. Vassanji, and D. J. Rowe, Nucl. Phys. A452, 240 (1986).

[25] O. Castaños, J. P. Draayer, and Y. Leschber, Z. Phys. A 329, 33 (1988)

[26] D. J. Rowe, M. G. Vassanji, and J. Carvalho, Nucl. Phys. A504, 76 (1989).

[27] Evaluated experimental nuclear structure data ENSDF, edited by National Nuclear Data Center, Brookhaven National Labo- ratory, Upton, NY 11973, USA (last updated Apr. 24, 1998). Updated experimental data were obtained via telnet at bnlnd2.dne.bnl.gov.

[28] B. R. Mottelson and J. G. Valatin, Phys. Rev. Lett. 5, 511 (1960).

[29] D. J. Thouless, Nucl. Phys. 21, 225 (1960).

[30] D. J. Rowe, Rev. Mod. Phys. 40, 153 (1968). 